\title{
Study of Stability Loss of Cylindrical Shell Using Experimental and Numerical Methods
}

\author{
Jozef Bocko, Ján Kostka*, Peter Pavelka, Tomáš Kula, Peter Čarák, Cosmin Stefan Roszkos, Patrik \\ Varga
}

Technical University of Košice, Faculty of Mechanical Engineering, Institute of Design Machine and Process Engineering, Department of Applied Mechanics and Mechanical Engineering, Letná 9, 04200 Košice, Slovak Republic

\begin{abstract}
The contribution of the paper is to determine the critical compressive force for a thinwalled cylindrical shell made of aluminium alloy. The solution of the problem involves use of the experimental method. A numerical approach, based on the finite strip method is chosen as the verification step. In order to determine the critical force, specimens representing a cylindrical thin-walled shell are used. Subsequently, the results obtained by the finite strip method are checked by experimental measurements.
\end{abstract}

Keywords: cylindrical shell, loss of stability, experimental measurement, critical force, finite strip method.

\section{Introduction and Historical Overview of Mathematical Theories}

Nowadays, thin-walled shell elements represent an optimal solution concept in a requirement where the main parameter is a high load-bearing capacity with a relatively low weight of the proposed structure. Such types of solutions can be applied in the field of civil engineering, aeronautics and general engineering. One of the negative phenomena connected with these structures is loss of stability as a result of compress loads. A particular problem with cylindrical shell elements is the determination of critical force causing loss of stability. The results gained for such structures are very sensitive to geometric deviations, the manner of shell fixation and to various imperfections of unknown origin. For this reason, several mathematical theories describing this negative phenomenon have been derived. The most famous works are listed below.

One of the first significant works based on the concept of linear elasticity was developed by Love [1]. In this work he started from Kirchhoff's hypotheses, which were originally derived for the theory of plate bending. By simplifying stress-strain and constitutional relationships, Love presented a set of mathematical solutions for thin-walled, flexible shell elements. Subsequent development [1], managed to remove some of the shortcomings contained in the mentioned mathematical theory. Reissner presented in his work [2], new equations of equilibrium as well as relations for deformations and stresses. By applying the principles of virtual work and Kirchhoff-Love hypotheses, the first order approximation theory was derived by Sanders [3]. Koiter introduced optimization of inconsistencies for relationships describing the twist of shells [4] and [5]. The authors [6], [7] and [8] independently developed an approximation theory of second order for shell elements. The general relations and equations of this theory are a direct result of the application of Kirchhoff's hypotheses together with the assumption of small displacements to the corresponding equations of the theory of elasticity. The application of this theory is generally limited to cylindrical shells. In addition, it was found that general relations of the second order approximation theory 
of shells are very difficult for the applicability of the calculation. Novozhilov at [9] developed another method of second order approximation theory. As with the theory based on [6], [7] and [8], relations for proportional deformations and stresses were obtained by applying the Kirchhoff hypotheses. Gol'denveizer has made valuable contributions to the general theory of thin shells [10]. He was the first who formulated the compatibility conditions for stress components in the general shell theory. Second order approximation equations were derived by Vlasov [11], directly from the general three-dimensional linear elasticity equations for the thick-walled shell. It has been hypothesized that the transverse normal and shear stress components may be neglected for thin-walled shells [12] The above mentioned theories were formulated from the classical shell linear theory. It is known that the equations of these theories, which are based on Hooke's law and neglect of non-linear terms in equations for stress components, and in equations of equilibrium, have a unique solution in each case. In other hands, linear shell theories determine a unique equilibrium position for each shell with a defined load and bond. Reissner [13] derived a non-linear theory of symmetrically loaded axisymmetric shells. In this theory, the assumption of small displacements has been abandoned, while the remaining assumptions of general higher order approximation theories have been retained. The derivation of the general geometric non-linear theory of thin shells was described by Naghdi and Nordgren [14], Sanders [15] and Koiter [16]. Vlasov at work [11] derived a set of equations for non-linear shells with a small radius of curvature. Subsequent development of the general nonlinear theory of thin shells was carried out by Mushtari and Galimov [17], Simmonds and Danielson [18].

\section{Proposal experimental and numerical Solutions}

The object of the study is a thin-walled cylindrical specimen of circular cross-section made by aluminium alloy. The designation $L$ represents the length, $h$ is the surface thickness and $r$ the radius of the cylinder, Figure 1. The dimensions of the specimen are:

$$
\begin{aligned}
\checkmark L & =100 \mathrm{~mm}, \\
\checkmark h & =0.11 \mathrm{~mm}, \\
\checkmark r & =33 \mathrm{~mm} .
\end{aligned}
$$
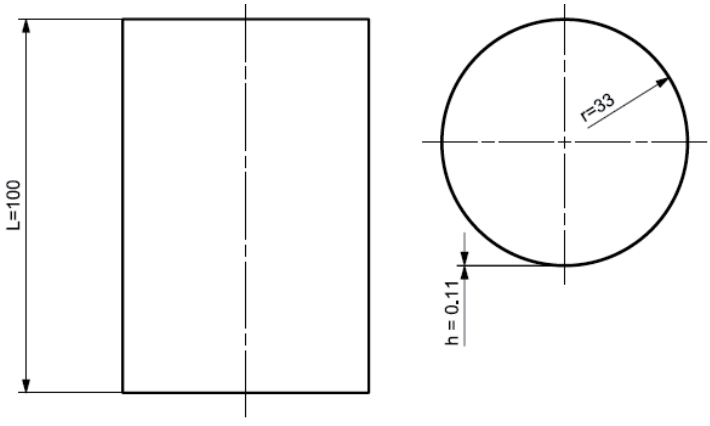

Figure 1: Dimensions of the test specimen.

In terms of length, cylindrical shells can be divided into three classes. The first class of the shells, are named, the shells of small lengths. Their specific stability loss behavior can be compared to the stability loss behavior of plates. The collapse of such shells usually results in only one sinusoidal halfwave in the axial direction, under the effect of axial compressive stress. The next one class are named long shells, whose character of loss of stability manifests itself as a displacement of the shell in the radial direction without local deformation of the surface also known as Euler's task of the pressured rod. From the standpoint of loss of stability, shells are referred to as cylindrical shells of medium lengths. Their collapse under the influence of compressive loads can cause local or global loss of stability. An example of a local loss of stability of a cylindrical shell may be the geometric imperfection of the shell which causes a deviation from the middle surface of the shell. Such slight deviations result in a significant decrease in shell bearing capacity and are therefore the most demanding in terms of structural design of medium-length shell [12], [19] and [20].

The relationship that characterizes the proposed shell into the above classes has the form [20]

$Z=\frac{L^{2}}{r h} \sqrt{\left(1-\varepsilon^{2}\right)}$,

where, $\varepsilon$ represents the Poisson ratio and $Z$ represents the shape factor. It is value of the ratio of shell length to its radius and is useful for distinguishing between short, medium and long cylindrical shells. If $Z$ magnitude is

$$
Z \leq 2.85 \text {, }
$$

then, shells are classified into short lengths class. On the other hand, if $Z$ magnitude is

$Z \geq 2.86$, 
then, shells are classified into medium and long lengths classes.

By solving the form (1) the value of $Z$ has been achieved $Z=2600$. Based on form (3), it's possible to determine correct class of shells length. The proposed test specimens may be considered to be shells of medium and long length [20]. In Figure 2, are given the test specimens.

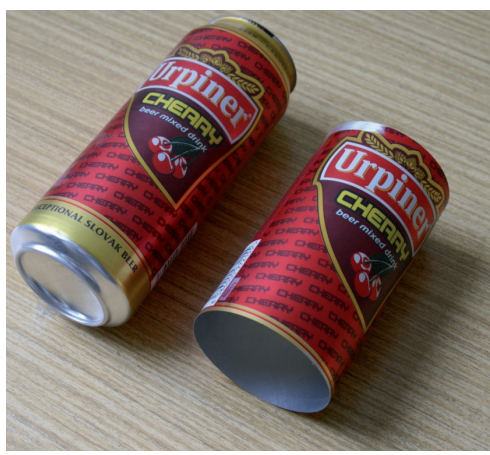

Figure 2: The test specimens.

The static pressure experimental test is carried out on a testing device. Both ends of the test sample are inserted into the fixtures, Figure 3, which is attached to the lower and upper jaws of the test device. In this way, unwanted displacement of the sample during loading is avoided, and due to the manufacturing tolerances of the fixtures, fixed bond of the shell can be assumed.

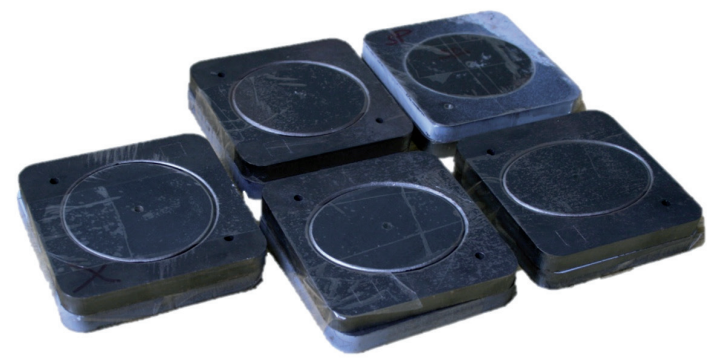

Figure 3: Variations of the fixtures.

20 pieces of test specimens were used for the experimental measurements, which is a sufficient sample for statistical evaluation of the measured data. The monitored statistics data are the standard deviation, arithmetic mean and median. A numerical calculation program based on the finite strip method was created to verify the obtained results. Before the numerical calculation itself, it was necessary to obtain material characteristics of the aluminium alloy used for these specimens.

\subsection{Proposal of the static tensile test}

The most commonly used method for the determination of basic material parameters is the static tensile test. In order to carry out such a test, it is necessary to produce test specimens intended for the tensile test according to the actual valid technical standard. In order to evaluate the tensile test data, the measurement must be repeated. The minimum recommended number of tensile test measurements is given by the standard. For this case a minimum of 10 measurements is recommended [21]. In the static tensile test evaluation process, samples whose breakage has occurred outside the functional area of the specimens should be excluded. In Figure 4, probable variants of sample breakage are shown.

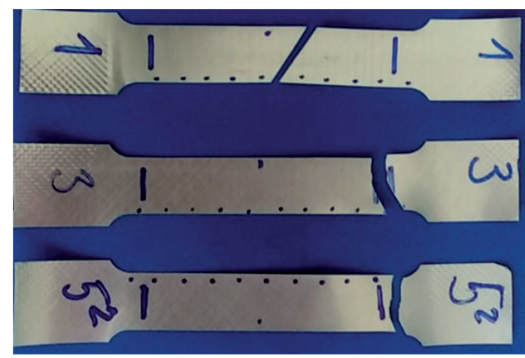

Figure 4: Variants of the specimens breakage.

The specimen labeled by 1 , represents the breakage point of the specimen in the functional area and such a sample is satisfactory for evaluation process. The specimen labeled by 3, represents the limit breaking point. It may be noted that the rupture passes through the defined functional area of specimen. Such cracked specimens may still be considered satisfactory for evaluation process, but account should be taken of the circumstances of fracture propagation. At last, the specimen labeled by $5^{2}$, is unsatisfactory due to the fact, that fracture is outside of the functional part of the specimen.

\subsection{Proposal of numerical program based on the finite strip method}

The finite strip method can be considered as a special form of the finite element procedure using the displacement approach. Unlike the standard finite element method, which uses polynomial displacement functions in all directions, the finite strip method calls for use of simple polynomials in some directions and continuously differentiable smooth series in the other directions, with the stipulation that such series should satisfy a priori 
the boundary conditions at the ends of the strips or prisms. The general form of the displacement function is given as a product of polynomials and series. Thus for a strip, in which a two-dimensional problem is reduced to a one-dimensional problem, [23]

$$
w=\sum_{m=1}^{r} f_{m}(x) Y_{m} .
$$

Similarly, in the case of a solid element (e.g. prism, cube, etc.), the three-dimensional problem is reduced to two-dimensional, and the displacement function may have the form

$$
w=\sum_{m=1}^{r} f_{m}(x, y) Y_{m} .
$$

Finally, the three dimensional problem is considered to be one dimensional in the case of layers if

In the above expressions, the series has been

$$
w=\sum_{m=1}^{r} \sum_{n=1}^{t} f_{m n}(z) X_{m} Y_{n} \text {. }
$$

simplified to the $r$ th, and $t$ th terms. $f_{m}(x), f_{m}(x, y)$ and $f_{m n}(z)$ are polynomial expressions with unspecified constants for $m$ th, $n$th order of the sum. $X_{m}, Y_{n}$ represent sums that satisfy the boundary conditions in the $x$, and $y$ directions and also indicate the deviated shapes in these directions. The final strip form is shown in Figure 5.

In the case of the proposed numerical program, derived stiffness matrices were used for the case, when the shell has at both ends by fixed-fixed boundary conditions [24].

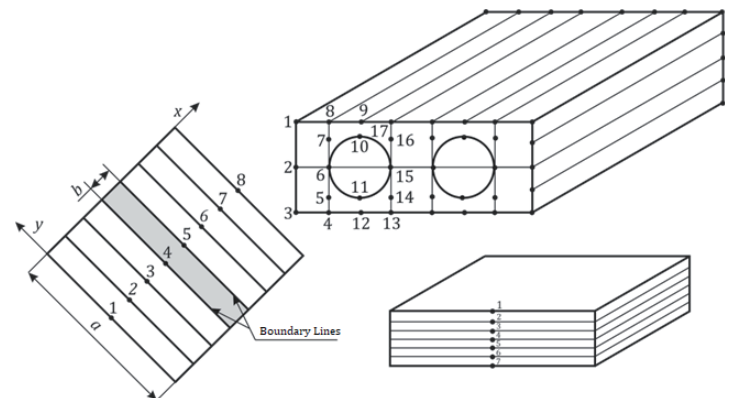

Figure 5: Form of the finite strip [23].

Table 1: Measured critical forces.

\section{Results}

\subsection{Result of statics compressive experimental measurement for critical force determination}

In the evaluation process of the experimenta measurement, 3 pieces of samples were not used. These samples were characterized by significantly lower achieved levels of critical force, than that of the other samples. This was caused by a spreading deformation from the fixture area of the samples, which was probably due to a poor stabilization of the specimen in the fixture, damage of the test specimen surface due to handling operations or unknown factors. The determined levels of the critical forces are listed in.

In the Table 2, statistical values of measured values of critical forces are given.

Table 2: Statistically processed and evaluated results of the critical forces.

\begin{tabular}{|l|l|l|}
\hline Standard deviation [-] & Arithmetic mean [N] & Median [N] \\
\hline 120.244 & 805.8 & 783.58 \\
\hline
\end{tabular}

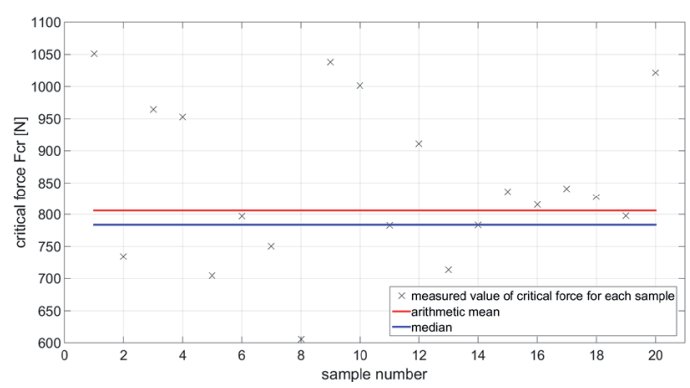

Figure 6: Critical forces (arithmetic mean and median).

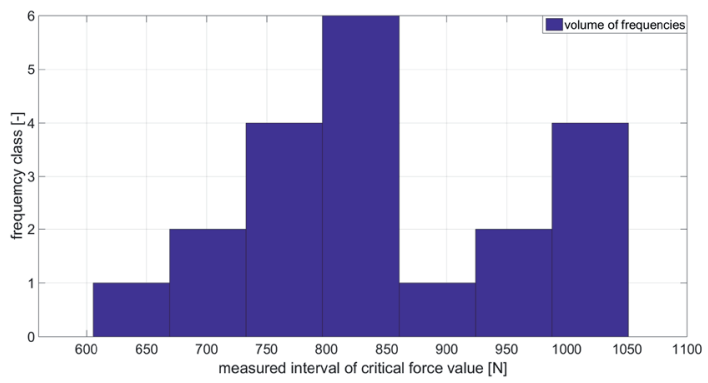

Figure 7: Histogram and normal distribution of determined critical forces.

\begin{tabular}{|l|l|l|l|l|l|l|l|l|l|l|}
\hline Sample number & 1 & 2 & 3 & 4 & 5 & 6 & 7 & 8 & 9 & 10 \\
\hline$F_{c}[\mathrm{~N}]$ & 1051.3 & 734.2 & 964.3 & 952.6 & 704.4 & 796.9 & 750.6 & 605.4 & 1038.2 & 1001.6 \\
\hline Sample number & 11 & 12 & 13 & 14 & 15 & 16 & 17 & 18 & 19 & 20 \\
\hline$F_{c}[\mathrm{~N}]$ & 782.5 & 910.6 & 713.6 & 783.5 & 836.2 & 815.3 & 840.9 & 827.6 & 797.4 & 1021.4 \\
\hline
\end{tabular}


The determined critical forces are plotted with arithmetic mean, median in Figure 6. In Figure 7, shows the constructed histogram along with the normal distribution of the measured values.

\subsection{Result of static tensile Test}

The determined tensile test parameters are:

$\checkmark E$ - Young modulus GPa,

$\checkmark F_{\max }-$ maximum achieved force $\mathrm{N}$,

$\checkmark \sigma_{m}$ - strength $\mathrm{MPa}$,

$\checkmark \varepsilon_{B}$ - elongation at fracture of sample, $\varepsilon_{M}$ - total strain at $F_{\max } \%$,

$\checkmark \sigma_{y}$-stress at yield point $\mathrm{kPa}$.

Table 3 shows the individual monitored variables for the respective samples. From the obtained tensile test data, it is possible to evaluate the Young modulus $E$, which is given in Table 4.

Further, we can compute shear modulus $G$. The form which is calculated is defined as

$$
G=\frac{E}{2(1+\varepsilon)} .
$$

The Poisson ratio has a value for aluminium alloys $\varepsilon=0.33$ [23].

Table 4: Statistically processed and evaluated data for Young modulus $\mathrm{E}$.

\begin{tabular}{|l|l|l|}
\hline Standard deviation [-] & Arithmetic mean [GPa] & Median [GPa] \\
\hline 5.448 & 20.53 & 21.39 \\
\hline
\end{tabular}

The Figure 8 shows histogram for the determined values of the Young modulus $\mathrm{E}$.

\subsection{Result of numerical calculation based on the finite strip method}

The material data based on the static tensile test entering the numerical calculation program are:

$\checkmark E=20.53 \mathrm{GPa}$,

$\checkmark G=7718 \mathrm{MPa}$,

Table 3: Determined values of static tensile test.

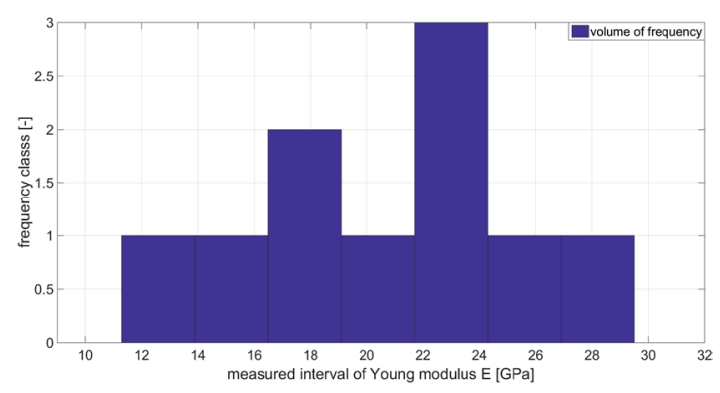

Figure 8: Histogram and normal distribution of determined Young modulus $\mathrm{E}$.

$\checkmark \varepsilon=0.33$.

The computed value of the critical force $F_{c r}$, determined by the finite strip method, is of the cylindrical shell.

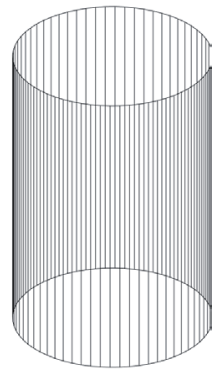

Figure 9: Mesh of finite strips of cylindrical shell.

Figure 10 shows collapsed shape of thin-walled cylindrical shell computed by the finite element method.

\section{Discussion}

As mentioned in the aforementioned papers [12], [19] and [20], there are three theoretical different values of critical loads. The first of the loads (9)

\begin{tabular}{|l|l|l|l|l|l|l|}
\hline Specimen number & $E[G \mathrm{~Pa}]$ & $F_{\max }[\mathrm{N}]$ & $\sigma_{m}[\mathrm{MPa}]$ & $\varepsilon_{B}[\%]$ & $\varepsilon_{M}[\%]$ & $\sigma_{y}[\mathrm{kPa}]$ \\
\hline 1 & 23.107 & 399.145 & 290.287 & 2.522 & 2.482 & 20381 \\
\hline 2 & 24.661 & 402.695 & 292.869 & 3.335 & 2.977 & 15280 \\
\hline 3 & 29.507 & 376.41 & 279.753 & 3.814 & 3.297 & 91567 \\
\hline 4 & 24.053 & 379.15 & 275.745 & 3.069 & 3.007 & 89574 \\
\hline 5 & 19.685 & 408.82 & 297.324 & 3.386 & 3.247 & 11763 \\
\hline 6 & 17.479 & 410.595 & 298.615 & 3.566 & 3.517 & 11705 \\
\hline 7 & 23.660 & 390.28 & 283.840 & 2.543 & 2.455 & 11705 \\
\hline 8 & 11.293 & 379.857 & 276.273 & 2.784 & 2.517 & 11469 \\
\hline 9 & 14.603 & 398.26 & 289.644 & 3.074 & 3.065 & 11996 \\
\hline 10 & 17.336 & 391.085 & 284.425 & 2.714 & 2.71 & 11469 \\
\hline
\end{tabular}



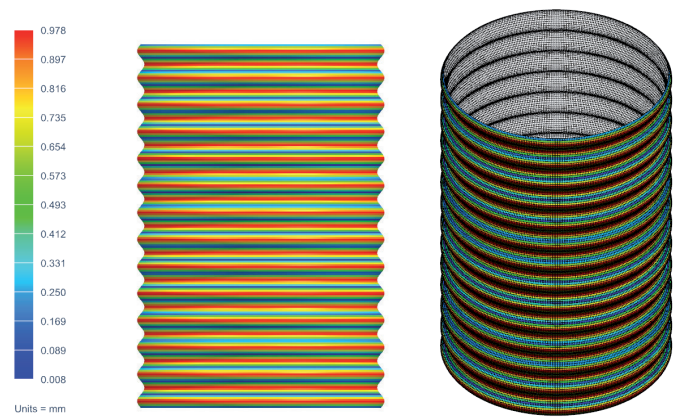

Figure 10: Collapsed shape of cylindrical shell determined by the finite element method.

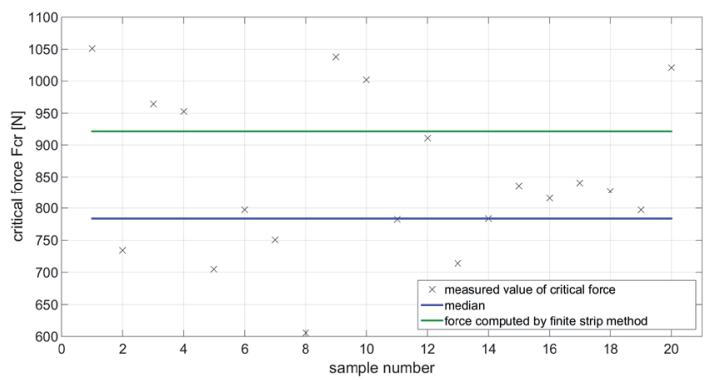

Figure 11: Graphically recorded determined critical forces and computed value using the FSM.

represents the state where the upper critical load can be defined as the largest load to which the original shell equilibrium configuration remains stable. The second of these loads is (10), which represents the lower critical load into which the original shell equilibrium configuration of the shell remains stable with respect to minimal surface damage. The third load $F_{c r}$ represents the actual stress value at which the cylindrical shell collapses. Their range is always between (9) and (10). For quality produced shells is the value of $F_{c r}$ closer to (9). The test samples were made from aluminium alloy can for storing carbonated beverages. Such storage containers are manufactured by deep drawing technology, which results in the initial of plastic deformations into the container shell, which create different stresses in the materials. This is one of the main reasons why the experimental measurement of critical forces resulted in a large dispersion of the measured values, which are shown in Table 1 and Table 2. The maximum and minimum values of critical force were measured for the sample with number 1 and number 8 . Their values are $F_{c r}^{\max }=1051.3 \mathrm{~N}$ and $F_{c r}^{\min }=605.4 \mathrm{~N}$, which represents a $42.4 \%$ decrease of critical force value. The calculated value of critical force using the finite strip method is

The difference between the finite strip method and the calculated median of the experimental measurement is $14.9 \%$. Figure 11 shows the determined critical force values obtained by experimental measurement and computed using the finite strip method.

\section{Conclusions}

The presented article introduces the historical development of mathematical theories, which are used for design of shell elements. In the second followed section (Proposal experimental and numerical Solutions) was described the individual tasks that had to be done before the experimental and numerical solution of the mentioned issue. A numerical method based on the finite strip method was used for the verification process. In terms of manufacturing accuracy, which was achieved by the manufacturing technology applied for the manufacture of fixtures, the fixtures could be considered fixed bond, which was also reflected in the boundary conditions and individual stiffness matrices of the proposed computational program. In order to consider the calculated values of the script correct, it was necessary to ensure the most accurate input material data. These were obtained by static tensile testing. The cause of the dispersion of the measured values of the critical forces is considered to be the initial plastic deformation of the casing, which arose as a result of manufacturing technological operations of the semi-product from which the test samples were, produced as well as shape imperfection. The difference between the verification calculation and the median of the measured forces is approx. 15\%. Such a significant difference in the determined values just makes the thin-walled shells more sensitive to different surface imperfections. This is the main reason why the proposed shell elements need to be manufactured in the required quality and accuracy class to ensure the highest possible real load-bearing capacity of the shell.

\section{Acknowledgments}

\section{The paper was created as part of the project VEGA 1/0500/20.}

\section{References and Notes}

1. Love, A. E. H. (1892/1944): A Treatise on the Mathematical Theory of Elasticity. Cambridge University Press, Dover.

2. Reissner, E (1941): A New Derivation of the Equations of the 
Deformation of Elastic Shells. Am. J. Math. Vol. 63, No. 1, pp. 177-184.

3. Sanders, J. L. (1959): An Improved First Approximation Theory for Thin Shells. NASA-TR-R24.

4. Koiter, W. T. (1960): A Consistent First Approximation in the General Theory of Thin Elastic Shells. Proc. Symposium on Theory of Thin Elastic Shells, pp. 12-33.

5. Ventsel, E., Krauthammer, T. (2001): Thin Plates and Shells Theory, Analysis and Applications. Marcel Dekker.

6. Lur'ye, A. I. (1940): General Theory of Elastic Shells. Prikl. Mat. Mekh., Vol. 4, No. 4, pp. 7-34.

7. Flügge, W. (1962): Stresses in Shells. Springer-Verlag, Berlin.

8. Byrne, R. (1944): Theory of Small Deformations of a Thin Elastic Shell. Math. N. S., Vol. 2, No. 1, pp. 103-152.

9. Novozhilov, V. V. (1964): Theory of Thin Elastic Shells. Noordhoff Groningen.

10. Gol'denveizer, A. L. (1961): Theory of Elastic Shells. Pergamon Press, New York.

11. Vlasov, V. Z. (1964): Theory of Shells and Its Applications in Engineering. NASA-TT-F-99, NASA, Washington, D.C.

12. Reddy, J. N. (2007): Theory and Analysis of Elastic Plates and Shells. CRC Press, New York.

13. Reissner, E. (1950): On Axisymmetrical Deformation of Thin Shells of Revolution. Proc. Symp. Appl. Math., Vol. 3, No. 1, pp. 27-52.

14. Naghdi, P. M., Nordgren, R. P. (1963): On the Nonlinear Theory of Shells under the Kirchhoff Hypothesis. Appl. Math., Vol. 21. No. 1, pp. 49-60.

15. Sanders, J. L. (1963): Nonlinear Theories for Thin Shells. Appl. Math., Vol. 21. No. 1, pp. 21-36.

16. Koiter, W. T. (1970): The Stability of Elastic Equilibrium. Diss. Tech. Rept. AFFDL-TR-70-25, Air Force System Command, Wright Patterson, AFB.

17. Mushtari, Kh. M, Galimov, K. Z. (1961): Nonlinear Theory of Thin Shells. The Israel Program for Scientific Translations, Jerusalem.

18. Simmonds, J. G, Danielson, D. A. (1972): Nonlinear Shell Theory with Finite Rotation and Stress-Function Vectors. Journal of Applied Mechanics, Vol. 39, pp. 1085-1090.

19. Donnell, H. L. (1976): Beams, Plates and Shells. McGraw-Hill, USA.

20. Narayanan, R. (1985): Shell Structures, Stability and Strength. Elsevier, London.

21. International Standard ISO 6892-1.: Metallic Material - Tensile Testing - Part 1: Method of Test at Room Temperature. Reference number ISO 6892-1:2009(E), First edition, ISO 2009.

22. Trebuňa, F., Šimčák, F. (2007): Príručka experimentálnej mechaniky. TypoPress, Košice.

23. Cheung, Y. K.: Finite Strip Method in Structural Analysis.
Paramount Press, Hungary.

24. Schafer, B. W., Ádány, S. (2006): Buckling Analysis of ColdFormed Steel Members Using CUFSM: Conventional and Constrained finite strip methods. 18th International Specialty Conference on Cold-Formed Steel Structures: Recent Research and Developments in Cold-Formed Steel Design and Construction. Orlando-Florida, 